\title{
From Notes to Vowels: Neural Correlations between Musical Training and Speech Processing
}

\author{
Iliza M. Butera \\ Neuroscience Graduate Program, Vanderbilt University, Nashville, Tennessee 37232 \\ Review of Bidelman and Alain
}

Both vocal and instrumental musical training put significant demands on auditory processing to resolve minute changes in timbre, timing, and pitch of both individual notes and their higher syntactic organization into melodies. Distinguishing timbres-the complex spectral and temporal acoustic properties that allow for differentiation of instruments playing the same note-is both crucial and challenging in complex arrangements like a full orchestra. Likewise, it is essential to establish precise timing through a keen awareness of temporal changes, such as the onset and offset of notes that guide musical rhythm and tempo. Lastly, acute perception of pitch is important for ordering notes as relatively higher or lower in frequency. An approximate 6\% deviation in frequency corresponds to one musical semitone that, for Western musicians, can mean the difference between playing in or out of key. On a violin, for instance, one semitone corresponds to a physical spacing on the order of millimeters, thereby requiring both fine pitch discrimination and excellent sensorimotor coordination. These skills are particularly evident in techniques like vibrato,

Received March 20, 2015; revised April 15, 2015; accepted April 17, 2015. This work was supported by a "Fundamentals in Neuroscience" training grant (T32 MH064913). I thank Mark Wallace, Reyna Gordon, Mike Butera, and Anita Disney for helpful comments and editing.

The author declares no competing financial interests.

Correspondence should be addressed to lliza Butera, Vanderbilt Brain

Institute, Vanderbilt University, 465 21st Avenue South, Nashville, TN 37232. E-mail: iliza.butera@vanderbilt.edu.

DOI:10.1523/JNEUROSCI.1102-15.2015

Copyright $\odot 2015$ the authors $\quad 0270-6474 / 15 / 358379-03 \$ 15.00 / 0$ where periodic motions create controlled fluctuations in the tuning of a single note.

Through frequent repetition, musicians develop enhanced auditory perceptual skills that are associated with experience-dependent structural and neurophysiological changes in the brain's response to sound. For example, the duration of piano training is correlated with the strength of neural activation by piano notes, indicating stronger cortical representations of these trained stimuli (Pantev et al., 1998). More recently, Hyde et al. (2009) correlated merely 15 months of instrumental musical training with structural brain changes in motor and auditory areas that paralleled behavioral improvements in motor and auditory musical tests. Moreover, these behavioral benefits can generalize to other forms of complex acoustic processing like speech (Parbery-Clark et al., 2009), which shares many spectrotemporal cues with music perception. For instance, vocal harmonics defining timbre can disambiguate the voices of multiple talkers, small timing differences in voice onset can cue phonetic distinctions, and pitch contours are important for discerning questions and statements as well as phonological meaning in tonal languages. Arguably, the transfer of musical proficiency to speech processing results from the higher demands that musical training places on each of these acoustic cues (Patel, 2014).

By noninvasively measuring eventrelated brain potentials (ERPs) through scalp-recorded EEG, researchers have shown shorter neural onset latencies and more robust cortical (Schön et al., 2004) and subcortical (Wong et al., 2007) encoding of speech in musicians compared with their non-musician peers. This holds true for both vocal and instrumental musical experience, and suggests that mechanisms of neuroplasticity linked to musical training may benefit speech and language processing through their shared auditory processing networks.

Previous studies by Bidelman et al. used a categorical phoneme perception task to investigate cross-domain neural enhancements in younger musicians (Bidelman et al., 2014a) and age-related neural deficits in non-musicians (Bidelman et al., 2014b). Other recent work has reported enhanced auditory brainstem responses of older musicians with hearing loss who had significantly less perceptual auditory decline (i.e., difficulty hearing in noise) compared with non-musicians with matched audiogram profiles (Parbery-Clark et al., 2013). What remained to be investigated was the resilience of auditory processing in musicians against age-related declines in auditoryevoked potentials before behavioral speech or hearing deficits. This question was addressed in Bidelman and Alain (2015) by measuring cortical and subcortical auditory-evoked potentials in musicians without hearing loss, most of whom were in their 60s and 70s. The control group consisted of age-matched adults with normal hearing sensitivity and $<2$ years of formal musical training in their lifetimes.

When presented with five vowel sounds with evenly spaced variants of 
their first formant frequency, subjects were instructed to phonetically categorize their percepts as /u/ or /a/. The key findings of this study are that musicians, compared with non-musicians, had (1) speeded neural onset latencies at the level of the subcortex and the cortex, (2) faster behavioral reaction times on the phonemic judgment, and (3) more separable neural representations (measured by P2 of the cortical ERP) when conditioned on the categorical percepts (Bidelman and Alain, 2015, their Fig. 5). These findings support an association between lifelong musical engagement and the resilient sharpening of auditory processing in older musicians.

In interpreting these results, Bidelman and Alain (2015) postulate that neuroplastic changes in auditory processing networks are not restricted by age. This implies the potential for late musical interventions to transfer benefits to speech processing, perhaps even at or near the onset of measurable age-related deficits. However, it is important to note that the subjects in this study began their musical training before the age of 14 , and it is possible that the timing of this training onset may have been critical for neuroplastic changes to ensue. That is, the structural changes underlying differential auditoryevoked responses late in life may have developed early in the musician group and remained stable for many years, thereby resisting age-related degradation rather than actively restructuring in response to deleterious effects of aging.

It is possible to address this issue, perhaps even within the current dataset, by investigating individual differences in the onset and amplitude of ERPs and their correlation with the onset and duration of musical training. For instance, a robust correlation with the duration of musical engagement could provide compelling evidence in support of continued, experience-dependent neuroplasticity such that continued musical engagement may be a stronger determinant of auditory-evoked potentials than early musical exposure. In future studies, the inclusion of individuals who began musical training later in life as well as those with early training that was discontinued in adolescence would provide valuable insight into this issue (as in Skoe and Kraus, 2012). Together, one could determine whether early, recent, and/or sustained training and practice is sufficient for robust auditory encoding.

It is also important to note that the results of Bidelman and Alain (2015) do not necessarily indicate causation between musical experience and auditory neuroplasticity. To reduce the possibility of other pre-existing factors influencing group differences (e.g., genetic and environmental), longitudinal studies have been conducted, primarily in younger children (Fujioka et al., 2006; Moreno et al., 2009; Francois and Schön, 2011; Chobert et al., 2014). In one example, Moreno et al. (2009) reported enhanced neural encoding of frequency shifts in music listening between groups of children randomly assigned to either a music class or painting class for 9 months. The physiological and behavioral benefits of such music training programs in children, paired with more recently described enhanced auditory/language skills in adult musicians, suggest similar randomized studies in older individuals should be conducted to directly assess the impact of continued or late music intervention programs.

Mechanisms of music-driven auditory plasticity are not yet well understood, but may involve the modulation of brainstem encoding via top-down corticofugal projections. This is supported by the selective enhancement of information-bearing acoustic features in the brainstems of musicians where, for example, upper harmonics that typically drive melodies have stronger neural representations as opposed to broad increases in gain across all frequencies (Lee et al., 2009; Kraus and Chandrasekaran, 2010). Indeed, corticofugal modulation may play a role in the strong group $\times$ vowel interaction of the cortical P3 amplitudes, both reported in Bidelman and Alain (2015) and widely associated with attention. These results suggest enhanced auditory reorienting in musicians that, taken together with recent work indicating attentional modulation of even cochlear sensitivity (Delano et al. 2007), provide support for models of feedback loops capable of modulating auditory afferent structures.

Other candidate mechanisms of plasticity include the increased establishment and/or strengthening of synapses at the level of the brainstem to fine-tune ascending sensory processing. For example, greater phaselocking of neurons to acoustic waveforms may encode a higher fidelity neural-acoustic representation through bottom-up plasticity that ultimately allows for the delivery of a more comprehensible signal to the cortex. In summary, it is worth noting that plasticity may be taking place at multiple spatial scales in both ascending and descending auditory pathways in ways that are not yet well described and warrant further investigation.

Finally, enhancements in higher cognitive functions like focused auditory attention and working memory can also shape auditory skills in musicians and benefit the understanding of speech in noise (Kraus et al., 2012; Anderson et al., 2013). Furthermore, deficits in both auditory working memory and attention have been described in populations with hearing loss as well as cochlear implant users (Pisoni and Geers, 2000; Torppa et al., 2014). Measuring the functional impact of high-fidelity acoustic encoding requires sufficiently taxing the auditory system by lowering the signal-to-noise ratio or, in the case of cochlear implant users, vastly down-sampling sound into coarse cochlear stimulation. Creating noisier neural representations either acoustically or electrically can require more faithful brainstem representations for cortical pattern recognition to successfully parse and categorize semantic meaning. Further work investigating the impact of musical training on improving thresholds for ecological listening environments like hearing in noise tests (as in Parbery-Clark et al., 2009) may reveal measurable functional benefits to complement the findings of the speeded reaction times recorded in the present study.

In summary, Bidelman and Alain (2015) describe a compelling association between life-long musical training and temporally precise categorical encoding of speech, despite the risk of age-related speech deficits. Further work in this area may better define the impact of musical training late in life for aging individuals and clinical populations such as cochlear implant users who could greatly benefit from the effects of musicianship.

\section{References}

Anderson S, White-Schwoch T, Parbery-Clark A, Kraus N (2013) A dynamic auditory-cognitive system supports speech-in-noise perception in older adults. Hear Res 300:18-32. CrossRef Medline

Bidelman GM, Alain C (2015) Musical training orchestrates coordinated neuroplasticity in auditory brainstem and cortex to counteract age-related declines in categorical vowel perception. J Neurosci 35:1240-1249. CrossRef Medline

Bidelman GM, Weiss MW, Moreno S, Alain C (2014a) Coordinated plasticity in brainstem and auditory cortex contributes to enhanced categorical speech perception in musicians. Eur J Neurosci 40:2662-2673. CrossRef Medline

Bidelman GM, Villafuerte JW, Moreno S, Alain C (2014b) Age-related changes in the subcorticalcortical encoding and categorical perception of speech. Neurobiol Aging 35:2526-2540. CrossRef Medline

Chobert J, François C, Velay JL, Besson M (2014) Twelve months of active musical training in 8to 10 -year-old children enhances the preattentive processing of syllabic duration and 
voice onset time. Cereb Cortex 24:956-967. CrossRef Medline

Delano PH, Elgueda D, Hamame CM, Robles L (2007) Selective attention to visual stimuli reduces cochlear sensitivity in chinchillas. J Neurosci 27:4146-4153. CrossRef Medline

Francois C, Schön D (2011) Musical expertise boosts implicit learning of both musical and linguistic structures. Cereb Cortex 21:23572365. CrossRef Medline

Fujioka T, Ross B, Kakigi R, Pantev C, Trainor LJ (2006) One year of musical training affects development of auditory cortical-evoked fields in young children. Brain 129:25932608. CrossRef Medline

Hyde KL, Lerch J, Norton A, Forgeard M, Winner E, Evans AC, Schlaug G (2009) Musical training shapes structural brain development. J Neurosci 29:3019-3025. CrossRef Medline

Kraus N, Chandrasekaran B (2010) Music training for the development of auditory skills. Nat Rev Neurosci 11:599-605. CrossRef Medline

Kraus N, Strait DL, Parbery-Clark A (2012) Cognitive factors shape brain networks for auditory skills: spotlight on auditory working memory. Ann N Y Acad Sci 1252:100-107. CrossRef Medline

Lee KM, Skoe E, Kraus N, Ashley R (2009) Selective subcortical enhancement of musical intervals in musicians. J Neurosci 29:5832-5840. CrossRef Medline

Moreno S, Marques C, Santos A, Santos M, Castro SL, Besson M (2009) Musical training influences linguistic abilities in 8-year-old children: more evidence for brain plasticity. Cereb Cortex 19:712-723. CrossRef Medline

Pantev C, Oostenveld R, Engelien A, Ross B, Roberts LE, Hoke M (1998) Increased auditory cortical representation in musicians. Nature 392:811-814. CrossRef Medline

Parbery-Clark A, Skoe E, Kraus N (2009) Musical experience limits the degradative effects of background noise on the neural processing of sound. J Neurosci 29:14100-14107. CrossRef Medline

Parbery-Clark A, Anderson S, Kraus N (2013) Musicians change their tune: how hearing loss alters the neural code. Hear Res 302:121-131. CrossRef Medline

Patel A (2014) Can nonlinguistic musical training change the way the brain processes speech?
The expanded OPERA hypothesis. Hear Res 308:98-108. CrossRef Medline

Pisoni DB, Geers AE (2000) Working memory in deaf children with cochlear implants: correlations between digit span and measures of spoken language processing. Ann Otol Rhinol Laryngol Suppl 185:92-93. Medline

Schön D, Magne C, Besson M (2004) The music of speech: music training facilitates pitch processing in both music and language. Psychophysiology 41:341-349. CrossRef Medline

Skoe E, Kraus N (2012) A little goes a long way: how the adult brain is shaped by musical training in childhood. J Neurosci 32:1150711510. CrossRef Medline

Torppa R, Huotilainen M, Leminen M, Lipsanen J, Tervaniemi M (2014) Interplay between singing and cortical processing of music: a longitudinal study in children with cochlear implants. Front Psychol 5:1389. CrossRef Medline

Wong PC, Skoe E, Russo NM, Dees T, Kraus N (2007) Musical experience shapes human brainstem encoding of linguistic pitch patterns. Nat Neurosci 10:420-422. Medline 